\title{
Identification of a small mutation panel of coding sequences to predict the efficacy of immunotherapy for lung adenocarcinoma
}

Ying $\mathrm{Li}^{1+}$, Wenbin Jiang ${ }^{1 \dagger}$, Tianhao $\mathrm{Li}^{1}$, Mengyue $\mathrm{Li}^{1}$, Xin $\mathrm{Li}^{1}$, Zheyang Zhang ${ }^{1}$, Sainan Zhang ${ }^{1}$, Yixin Liu ${ }^{1}$, Wenyuan Zhao', Yunyan Gu', Lishuang $\mathrm{Qi}^{1{ }^{*}}$, Lu Ao ${ }^{2^{*}}$ and Zheng Guo ${ }^{1,2,3^{*}} \mathbb{D}$

\begin{abstract}
Background: Immune checkpoint inhibitors are effective in some cases of lung adenocarcinoma (LUAD). Wholeexome sequencing has revealed that the tumour mutation burden (TMB) is associated with clinical benefits among patients from immune checkpoint inhibitors. Several commercial mutation panels have been developed for estimating the TMB regardless of the cancer type. However, different cancer types have different mutational landscapes; hence, this study aimed to develop a small cancer-type-specific mutation panel for high-accuracy estimation of the TMB of LUAD patients.
\end{abstract}

Methods: We developed a small cancer-type-specific mutation panel based on coding sequences (CDSs) rather than genes, for LUAD patients. Using somatic CDSs mutation data from 486 LUAD patients in The Cancer Genome Atlas (TCGA) database, we pre-selected a set of CDSs with mutation states significantly correlated with the TMB, from which we selected a CDS mutation panel with a panel-score most significantly correlated with the TMB, using a genetic algorithm.

Results: A mutation panel containing 106 CDSs of 100 genes with only $0.34 \mathrm{Mb}$ was developed, whose length was much shorter than current commercial mutation panels of $0.80-0.92 \mathrm{Mb}$. The correlation of this panel with the TMB was validated in two independent LUAD datasets with progression-free survival data for patients treated with nivolumab plus ipilimumab and pembrolizumab immunotherapies, respectively. In both test datasets, survival analyses revealed that patients with a high TMB predicted via the 106-CDS mutation panel with a cut-point of 6.20 mutations per megabase, median panel score in the training dataset, had a significantly longer progression-free survival than those with a low predicted TMB $(\log -$ rank $p=0.0018, \mathrm{HR}=3.35,95 \% \mathrm{Cl} 1.51-7.42$; $\log$-rank $p=0.0020, \mathrm{HR}=5.06$, $95 \% \mathrm{Cl}$ 1.63-15.69). This small panel better predicted the efficacy of immunotherapy than current commercial mutation panels.

\footnotetext{
*Correspondence: qilishuang7@ems.hrbmu.edu.cn; lukey@fimu.edu.cn; guoz@ems.hrbmu.edu.cn

${ }^{\dagger}$ Ying Li and Wenbin Jiang contributed equally to this work

${ }^{1}$ College of Bioinformatics Science and Technology, Harbin Medical

University, Harbin 150086, China

2 Department of Bioinformatics, Key Laboratory of Ministry of Education

for Gastrointestinal Cancer, School of Basic Medical Sciences, Fujian

Medical University, Fuzhou 350001, China

Full list of author information is available at the end of the article
}

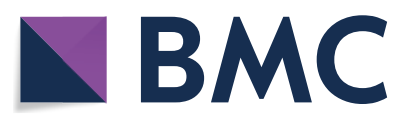

(c) The Author(s) 2020. This article is licensed under a Creative Commons Attribution 4.0 International License, which permits use, sharing, adaptation, distribution and reproduction in any medium or format, as long as you give appropriate credit to the original author(s) and the source, provide a link to the Creative Commons licence, and indicate if changes were made. The images or other third party material in this article are included in the article's Creative Commons licence, unless indicated otherwise in a credit line to the material. If material is not included in the article's Creative Commons licence and your intended use is not permitted by statutory regulation or exceeds the permitted use, you will need to obtain permission directly from the copyright holder. To view a copy of this licence, visit http://creativeco mmons.org/licenses/by/4.0/. The Creative Commons Public Domain Dedication waiver (http://creativecommons.org/publicdomain/ zero/1.0/) applies to the data made available in this article, unless otherwise stated in a credit line to the data. 
Conclusions: The small-CDS mutation panel of only $0.34 \mathrm{Mb}$ is superior to current commercial mutation panels and can better predict the efficacy of immunotherapy for LUAD patients, and its low cost and time-intensiveness make it more suitable for clinical applications.

Keywords: Immunotherapy, Tumour mutation burden, CDS mutation panel, Lung adenocarcinoma, Clinical application

\section{Background}

Lung adenocarcinoma (LUAD) is the most common type of non-small cell lung cancer (NSCLC), accounting for approximately $80-90 \%$ cases of lung cancer [1]. Currently, approximately $35-75 \%$ of LUAD patients relapse or die within 5 years of receiving conventional treatments based on the National Comprehensive Cancer Network Clinical Practice Guidelines in Oncology [2]. Recently, immunotherapies, which eliminate tumours by activating the immune system [3], have shown great promise for NSCLC $[4,5]$. For example, an immune checkpoint inhibitor, nivolumab, which targets programmed cell death protein-1 (PD-1), can significantly increase survival in advanced-stage NSCLC by blocking the interaction between PD-1 and its ligand programmed death-ligand 1 (PD-L1) and allowing cytotoxic T lymphocytes to act on tumour cells [6]. Furthermore, the inhibitor (ipilimumab) for cytotoxic $\mathrm{T}$ lymphocyte-associated antigen 4 (CTLA-4), which suppresses immune responses, has been approved for treating NSCLC [5] and some other cancers [7]. However, the heterogeneity of the response to immune checkpoint inhibitors significantly confounds the treatment of NSCLC [3]. Therefore, it is important to identify patients potentially benefiting from these immune checkpoint inhibitors.

Previously, PD-L1 protein expression in NSCLC patients has been approved as an auxiliary predictive marker for certain PD-1/PD-L1 inhibitors including pembrolizumab [8]. However, PD-L1 protein expression alone cannot completely account for the survival benefit to patients treated with immune checkpoint inhibitors [8-11]. Moreover, analysis of PD-L1 protein expression via immunohistochemistry (IHC) is challenging because of subjective diagnoses of immunostaining results by pathologists using different criteria or interpretations [12].

Several previous studies have reported a high tumour mutation burden (TMB), determined through wholeexome sequencing (WES), indicating that patients are more likely to harbour neoantigens, can predict the sensitivity to immunotherapies [13, 14]. For example, high-TMB patients are associated with enhanced responses to nivolumab (PD-1 inhibitor) plus ipilimumab (CTLA-4 inhibitor) immunotherapy [15].
Moreover, a high TMB is more significantly associated with the response to PD-1/PD-L1 inhibitors than with PD-L1 protein expression detected via IHC [16]. However, WES, necessary to determine the TMB, is not routinely performed in clinical practice because it is costly, time-consuming and labour intensive, and needs a large number of sequences [3, 17, 18]. Previous studies have reported that the TMB can be accurately estimated using smaller gene panels encompassing several hundred genes, such as the 324-gene mutation panel (FoundationOne CDxTM assay) [6, 19-21] and the 341-gene mutation panel (MSK-IMPACT) [22, 23], which have been clinically used. The cost-effectiveness of these mutation panels facilitates a greater sequencing depth than that of WES and consequently a higher ability to detect mutations, even for genes mutated in some tumour cells [24]. However, these commercial mutation panels were selected from cancer-related genes regardless of the cancer type, rather than being developed via a feature selection method; thus, mutation panels can still be improved. In particular, it is necessary to develop a cancer-type-specific mutation panel to estimate the TMB of LUAD samples, since different cancer types have different mutation landscapes [25]. Recently, Lyu et al. [3] constructed a LUAD-specific 24-gene model for predicting the TMB of LUAD samples. However, this panel was also based on complete exons of the panel genes, comprising thousands of exons in the panel genes, most of which being unmutated, solely increasing the unnecessary cost and time for sequencing.

In this study, based on the coding sequences (CDSs) with a high frequency of mutation in LUAD, we developed a CDS mutation panel to estimate the TMB of LUAD samples. Thereafter, we determined the correlation of CDSs in the mutation panel with the TMB in two independent datasets. From two datasets (Matthew and Rizvi), we used data on progression-free survival (PFS) of advanced LUAD patients treated with immune checkpoint inhibitors to estimate the performance of the CDS mutation panel for predicting the efficacy of immunotherapy. Furthermore, the CDS mutation panel was compared with two commercial mutation panels (324-gene and 341-gene panels) and a LUAD-specific mutation panel (24-gene panel). 


\section{Methods}

Data sources and pre-processing

The three LUAD somatic mutation datasets (Table 1) were used to construct and validate the mutation panel for estimating the TMB. The training mutation data were downloaded from The Cancer Genome Atlas (TCGA) database (https://portal.gdc.cancer.gov/), comprising 486 LUAD samples with paired mRNA expression data. For validation, we obtained two independent somatic mutation datasets with PFS data for patients treated with immune checkpoint inhibitors, including 59 LUAD samples reported by Matthew et al. [5] and 29 LUAD samples reported by Rizvi et al. [4]. The patients included in the Matthew dataset were treated with nivolumab (PD-1 inhibitor) plus ipilimumab (CTLA-4 inhibitor) and those in the Rizvi dataset were treated with pembrolizumab (PD-1 inhibitor) immunotherapy.

Whole-exome sequencing was previously performed for these TCGA data with tumour tissues and matched normal tissue or blood, which were used to filter out germline mutations and screen somatic mutations [26]; the

Table 1 Whole-exome sequencing mutation data analyzed in this study

\begin{tabular}{|c|c|c|c|}
\hline \multirow[t]{2}{*}{ Patient characteristics } & \multirow{2}{*}{$\begin{array}{l}\text { TCGA } \\
\text { No. (\%) }\end{array}$} & \multirow{2}{*}{$\begin{array}{l}\text { Matthew [5] } \\
\text { No. (\%) }\end{array}$} & \multirow{2}{*}{$\begin{array}{l}\text { Rizvi [4] } \\
\text { No. (\%) }\end{array}$} \\
\hline & & & \\
\hline \multicolumn{4}{|l|}{ Histology } \\
\hline Adenocarcinoma & 486 & 59 & 29 \\
\hline \multicolumn{4}{|l|}{ Age (years) } \\
\hline No less than 65 & $223(46)$ & $29(50)$ & $10(34)$ \\
\hline Less than 65 & $263(54)$ & $30(50)$ & $19(66)$ \\
\hline \multicolumn{4}{|l|}{ Sex } \\
\hline Male & $222(46)$ & $22(37)$ & $13(45)$ \\
\hline Female & $264(54)$ & $37(63)$ & $16(55)$ \\
\hline \multicolumn{4}{|l|}{ Smoking status } \\
\hline Never & - & $13(22)$ & $5(17)$ \\
\hline Former/light & - & $38(64)$ & $18(62)$ \\
\hline Current/heavy & - & $8(14)$ & $6(21)$ \\
\hline \multicolumn{4}{|l|}{ Stage } \\
\hline I & $263(54)$ & - & - \\
\hline$\|$ & $117(24)$ & - & - \\
\hline IIIA & $70(14)$ & - & - \\
\hline IIIB-IV & $36(7)$ & $59(100)$ & $29(100)$ \\
\hline \multicolumn{4}{|l|}{ PFS-status } \\
\hline Progression & - & $40(68)$ & $20(69)$ \\
\hline Progression-free & - & $19(32)$ & $9(31)$ \\
\hline \multicolumn{4}{|l|}{ Percentage of tumour cells } \\
\hline Known & $433(89)$ & - & - \\
\hline Unknown & $53(11)$ & - & - \\
\hline $\begin{array}{l}\text { Average percentage of } \\
\text { tumour cells }\end{array}$ & 78.76 & - & - \\
\hline
\end{tabular}

detailed protocol is described in the original literature [27]. Briefly, 0.5-3 $\mu \mathrm{g}$ of DNA from each sample was used for library preparation and sequenced using the Illumina HiSeq platform. The mean coverage across targeted bases on tumour and germline DNA were 97.63 and 95.83, respectively. Mutations with a variant allelic fraction of $<0.05$ in tumour tissue were excluded. Only the nonsynonymous mutations, including missense mutation, nonsense mutation, nonstop mutation, frame-shift mutation and in-frame mutation, were included, and a discrete mutation profile including 82,574 CDSs of 16,961 genes was generated. For the two test mutation data, wholeexome sequencing was performed for tumour tissues and matched normal tissues or blood. The detailed protocol is further described in the original literatures [5, 28]. Finally, discrete mutation profiles including 18,793 CDSs of 9400 genes and 8711 CDSs of 5504 genes were generated, wherein the CDSs mutation matrix was constructed using matched human reference genome annotated files derived from GENCODE (https://www.gencodegen es.org/human/releases.html).

\section{Development of the CDS mutation panel for estimating TMB}

First, from TCGA LUAD somatic mutation data, we extracted mutations in the CDSs using the human reference genome annotated file (GRCh38), and selected non-synonymous mutations to construct an $m^{*} n$ CDSs mutation matrix, where $m$ represents the number of CDSs in genes and $n$ represents the number of samples. TMB was estimated as (total mutations in CDSs/total bases of CDSs) $* 10^{6}$.

Thereafter, Spearman's rank correlation analysis was performed to estimate the correlation of the CDSs mutation state with the TMB. Herein, we restricted the analysis to the CDSs mutated in more than 5\% cancer samples $[29,30]$ to filter out 'passenger' genes with low-frequency mutations, as it may be subjected to random mutations rather than having a tumorigenic advantage. p-values were adjusted using the Benjamini-Hochberg procedure [31] for multiple testing to control the false discovery rate (FDR). CDSs significantly correlated with the TMB were selected as candidates.

Finally, the genetic algorithm (GA package) was used to generate a final CDS panel from among candidate CDSs, whose panel-score was most correlated with TMB. The genetic algorithm was implemented with a population size of 5000 and a crossover fraction of 0.9 ; it was terminated if the optimization objective of the best subset was not improved in 100 generations. Details regarding the genetic algorithm are shown in Additional file 1 . The correlation $\left(\mathrm{R}^{2}\right)$ was estimated via 
linear regression analysis [32]. Here, the panel-score was calculated as following (Formula 1):

$$
\text { Panel-score }=\beta \frac{\sum_{i=1}^{n} k_{i}}{l * 10^{-6}}+C
$$

where $n$ is the number of CDSs in the panel, $l$ is the length of the panel, and $k_{i}$ is the number of mutations in $i$-th CDS; $\beta$ and $C$ was obtained through linear regression analysis, $\beta$ is a coefficient to balance the panel-score and TMB, $C$ is a constant.

As no clinical data regarding immunotherapy were available for patients in TCGA, we could not determine the optimal cut-point for our CDS panel for predicting the efficacy of immunotherapy. Therefore, we set the cut-point of our CDS panel at a median panel score in TCGA.

\section{Survival analysis}

PFS was defined as the period during and after the treatment of a disease, wherein a patient lives with the disease but it is not exacerbated. The survival curve was estimated using the Kaplan-Meier method and compared using the log-rank test (survival package: 'survdiff') [33]. The univariate Cox proportional hazards regression model (survival package: 'coxph') was used to evaluate the predictive performances of the mutation panels. Furthermore, the multivariate Cox model (survival package: 'coxph') was used to evaluate the independent prognostic value of our CDS mutation panel after adjusting for clinical factors including age, sex, and smoking. Hazard ratios (HRs) and 95\% confidence intervals (CIs) were generated using the Cox proportional hazards model (survival package: 'coxph').

\section{Functional enrichment analysis}

Functional pathways for enrichment analysis were downloaded from Gene Ontology (GO) in November 2018. First, we performed Student's t-test with a 5\% FDR control to select differentially expressed genes (DE genes) between the high-TMB and low-TMB groups classified by the CDS panel. Here, 17,680 genes were used for differential expression analysis. Thereafter, the hypergeometric distribution model was used to determine whether the number of DE genes observed in a functional term was significantly greater than that expected through random chance.

All statistical analyses were performed by using $\mathrm{R}$ software packages version 3.4.2 (http://www.rproject.org/). Significance was defined as $p<0.05$ or $\mathrm{FDR}<0.05$ for multiple testing.

\section{Results}

\section{Construction of the CDS mutation panel}

Figure 1 provides a schematic representation of the study protocol.

From the LUAD mutation data from TCGA, we extracted mutation data from the CDSs by using the human reference genome annotated file (GRCh38). After selecting non-synonymous mutations, a mutation matrix comprising 82,574 CDSs and 486 patients was generated. Thereafter, using Spearman's rank correlation analysis, with a 5\% FDR control, 219 CDSs were significantly correlated with the TMB of the LUAD samples derived from TCGA data. Using the genetic algorithm ("Methods"), we extracted a CDS panel comprising 106 CDSs in 100 genes with a length of $0.34 \mathrm{Mb}$ (Additional file 2: Table S1), whose panel-score was most significantly correlated with the TMB of the LUAD samples $\left(\mathrm{R}^{2}=0.95\right.$, Fig. $\left.2 \mathrm{a}\right)$. This mutation panel was termed the 106-CDS panel. In the formula for the panel-score, $\beta=0.33, \mathrm{C}=0.27$ were obtained from linear regression analysis [32]. The technical details of the 106-CDS panel for TMB evaluation is described in Additional file 3: Table S2.

Furthermore, we estimated the correlation of the clinical factors and tumour cell proportions with the TMB using Spearman's rank correlation and found that only age and sex were significantly associated with the TMB (age: $p=0.0055$; sex: $p=0.0442$, Additional file 4: Table S3). Therefore, we additionally used the multiple linear regression model for the 106-CDS panel, age and sex together to estimate their correlations with the TMB. Consequently, the 106-CDS panel was still significantly correlated with the TMB, while age and sex were not, suggesting that the 106-CDS panel was an independent predictor of the TMB. Additionally, to prevent overestimating the TMB, since gene panels are usually heavily targeted at recurrently mutated genomic regions, we redetermined the TMB after excluding the mutations in cancer-related genes and the recurrent CDSs occurring in more than 5\% samples, and found that the correlation $\left(\mathrm{R}^{2}\right)$ of the 106-CDS panel with the recalculated TMB also approached 0.94 (Fig. 2b).

\section{Validation of the 106-CDS panel}

First, we applied the 106-CDS panel to 59 samples from the Matthew dataset with PFS data of patients receiving nivolumab plus ipilimumab immunotherapy. The correlation $\left(R^{2}\right)$ between the panel-score of the 106-CDS and the TMB was 0.82 (linear regression analysis, $p<0.0001$, Fig. 2c). When the panel score was dichotomized at 6.20 mutations per megabase (mut/Mb), the median of the panel-scores from the training dataset, our 106-CDS panel classified 18 and 41 patients into high- and low-risk 


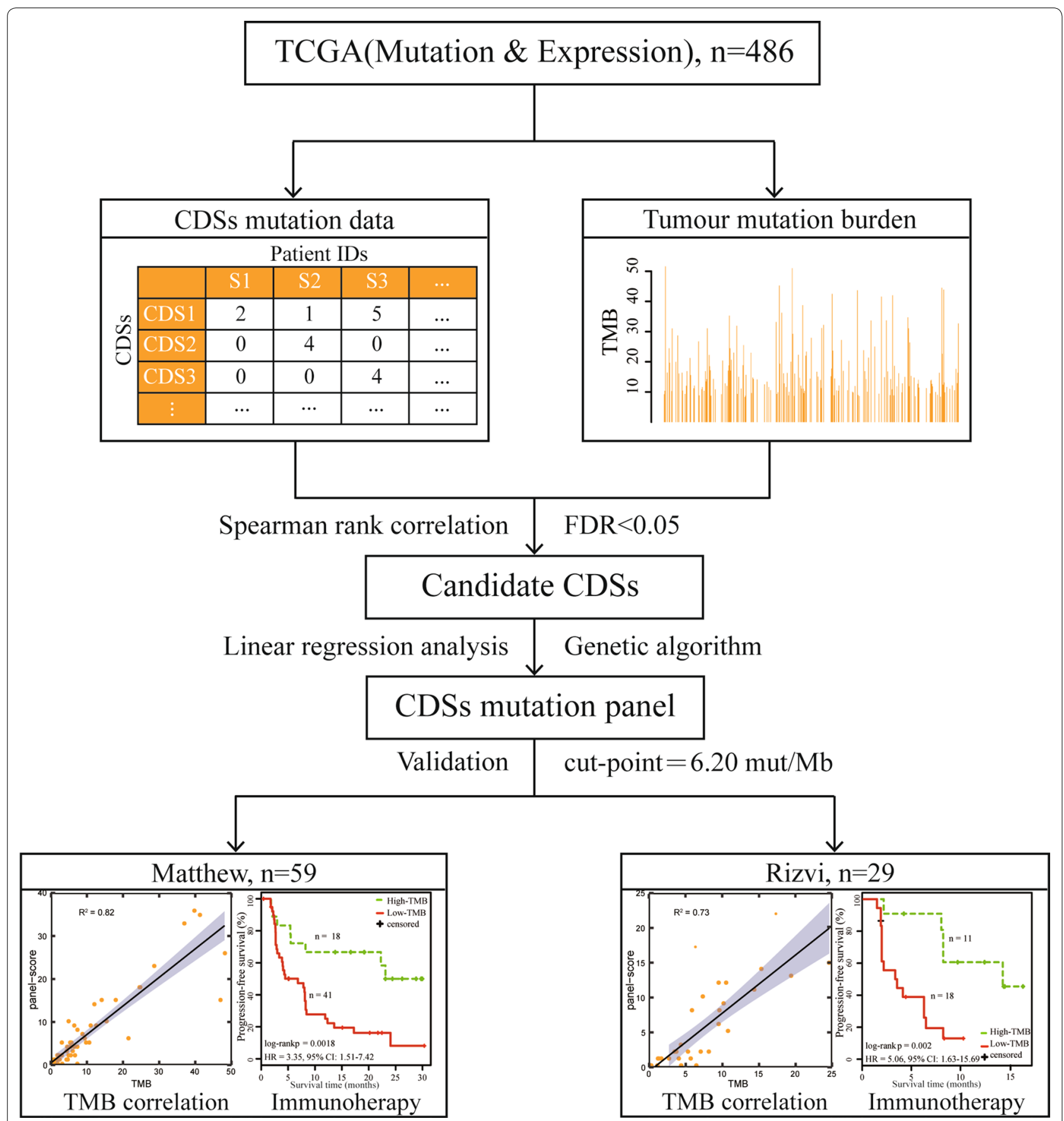

Fig. 1 Flowchart for the identification of the mutation panel. Mutation data for coding sequences (CDSs) in TCGA from 486 patients was used to develop a CDS mutation panel for estimating the tumour mutation burden. The performance of the CDS mutation panel was validated in two datasets with data on progression-free survival for patients treated with immune checkpoint inhibitors

groups, respectively. Univariate survival analysis revealed that the predicted high-TMB patients had significantly longer PFS than the predicted low-TMB patients (logrank $p=0.0018, \mathrm{HR}=3.35,95 \%$ CI 1.51-7.42, Fig. 3a). The 1-year PFS rate of the predicted high-TMB patients was 0.67 , which was markedly higher than the 1-year PFS rate $(0.25)$ of the predicted low-TMB patients. Multivariate Cox analysis revealed that the 106-CDS panel with a cut-point of $6.20 \mathrm{mut} / \mathrm{Mb}$ remained significantly associated with PFS $(p=0.0013, \mathrm{HR}=4.03,95 \%$ CI $1.73-9.40$, 

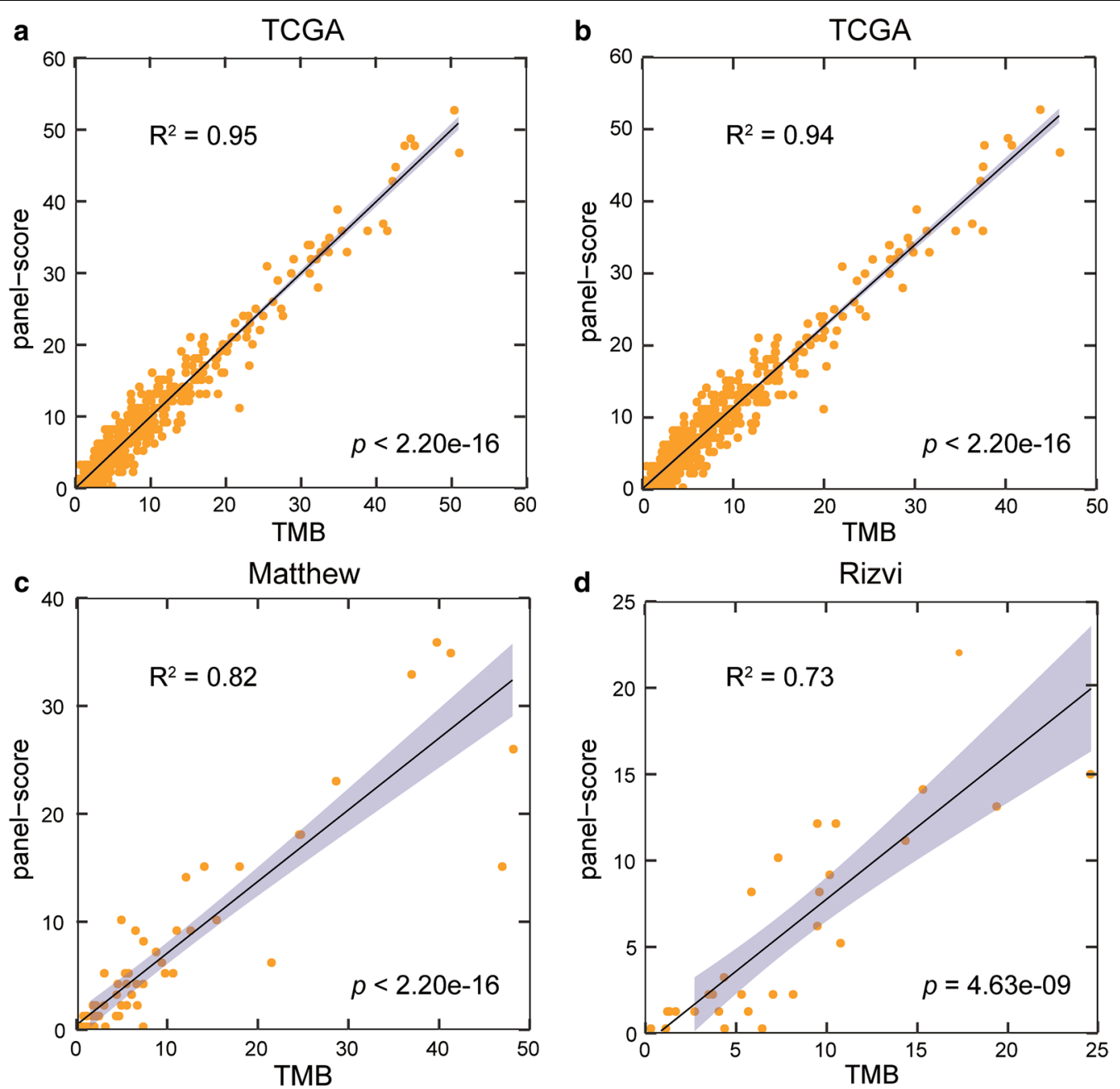

Fig. 2 Performance of the 106-CDS panel for the tumour mutation burden evaluation. a Linear regression analysis of the 106-CDS panel-score with the tumour mutation burden (TMB) of lung adenocarcinoma (LUAD) in TCGA database (training set). $\mathbf{b}$ Linear regression analysis of the 106-CDS panel-score with the recalculated TMB in TCGA after excluding the mutations in cancer-related genes and the recurrent CDSs occurring in more than $5 \%$ samples. $\mathbf{c}$ Linear regression analysis of the 106-CDS panel-score and the TMB in the Matthew dataset. $\mathbf{d}$ Linear regression analysis of the 106-CDS panel-score and the TMB in the Rizvi dataset. The gray lines are 95\% confidence intervals of the 106-CDS panel for the TMB evaluation

Fig. 3b) after adjusting for age ( $>65$ vs. $\leq 65$ years), sex (Male vs. Female), and smoking status (Current vs. Former vs. Never).

Similar results were obtained with the Rizvi dataset, wherein the correlation $\left(\mathrm{R}^{2}\right)$ between panel-score and the TMB was 0.73 (linear regression analysis, $p<0.0001$, Fig. 2d). High-TMB patients predicted using the 106CDS panel with a cut-point of $6.20 \mathrm{mut} / \mathrm{Mb}$ had a significantly longer PFS than the predicted low-TMB patients (log-rank $p=0.0020, \mathrm{HR}=5.06,95 \%$ CI 1.63-15.69, Fig. 3c). The 1-year PFS rate of the predicted high-TMB patients was 0.61 , markedly higher than the 1-year PFS rate $(0.13)$ of the predicted low-TMB samples. Multivariate Cox analysis revealed that the CDS panel with a cut-point of $6.20 \mathrm{mut} / \mathrm{Mb}$ remained significantly associated with PFS ( $p=0.0150, \mathrm{HR}=4.36,95 \% \mathrm{CI} 1.33-14.28$, Fig. 3d) after adjusting for age ( $>65$ vs. $\leq 65$ years), sex (male vs. female), and smoking status (current vs. former vs. never).

\section{Comparison of the 106-CDS panel with three mutation panels}

We compared our 106-CDS panel with two commercial mutation panels (324-gene [6, 20, 21] and 341-gene [23] panels) approved by the Food and Drug Administration (FDA), and a LUAD-specific mutation panel (24-gene panel) for predicting the efficacy of immunotherapy for 


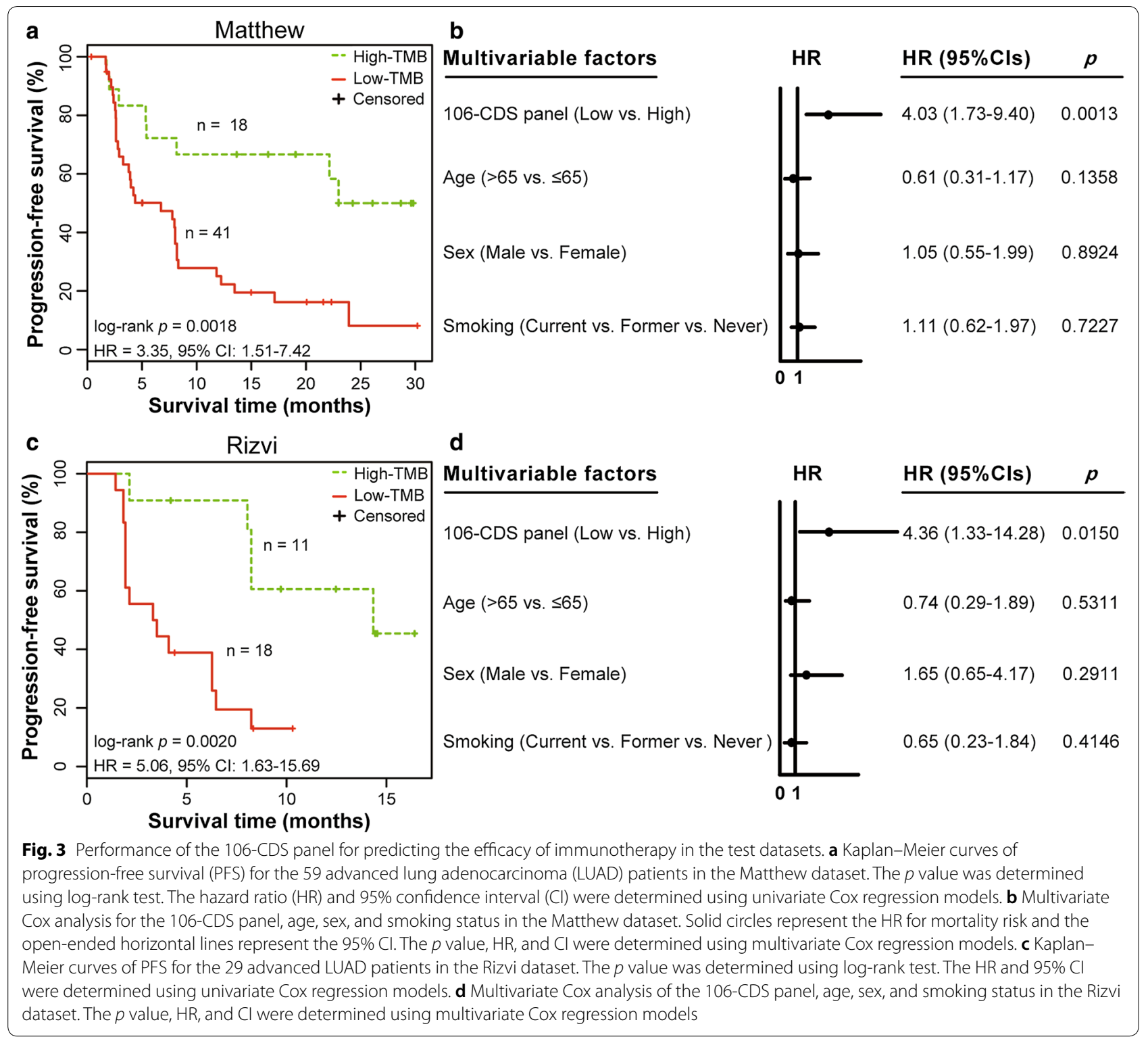

Table 2 The survival analysis result of all datasets

\begin{tabular}{lllll}
\hline Dataset & Mutation panels & Log-rank $\boldsymbol{p}$ & $\operatorname{Cox} \boldsymbol{p}^{\mathbf{a}}$ & $\begin{array}{l}\text { Hazard ratio (95\% } \\
\mathbf{C l s})^{\mathbf{a}}\end{array}$ \\
\hline Matthew & 106-CDS & 0.0018 & 0.0029 & $3.35(1.51-7.42)$ \\
& 324-gene & 0.0042 & 0.0057 & $2.65(1.33-5.28)$ \\
& 341-gene & 0.0135 & 0.0156 & $2.20(1.16-4.17)$ \\
& 24-gene & 0.0283 & 0.0312 & $2.04(1.07-3.89)$ \\
Rizvi & 106-CDS & 0.0020 & 0.0050 & $5.06(1.63-15.69)$ \\
& 324-gene & 0.0137 & 0.0208 & $3.74(1.22-11.46)$ \\
& 341-gene & 0.1233 & 0.1193 & $2.06(0.83-5.14)$ \\
\hline
\end{tabular}

a Cox $p$ value and Hazard ratio $(95 \% \mathrm{Cls}$ ) were generated by the univariate Cox proportional hazards model
LUAD. The results of univariate survival analyses are summarized in Table 2.

For the 324-gene mutation panel with a cut-point of $10 \mathrm{mut} / \mathrm{Mb}$ [19], containing $6130 \mathrm{CDSs}$ spanning $0.80 \mathrm{Mb}$, univariate survival analyses revealed that the two groups of patients classified using the panel had significantly different PFS after receiving immunotherapy in the Matthew dataset $(\log$-rank $\mathrm{p}=0.0042$, $\mathrm{HR}=2.65$, 95\% CI 1.33-5.28, Fig. 4a) and in the Rizvi dataset (log-rank $\mathrm{p}=0.0137, \mathrm{HR}=3.74,95 \%$ CI 1.22 11.46, Fig. 4b). However, univariate survival results revealed that its performance (HR) in predicting the efficacy of immunotherapy was worse than that of our 106-CDS panel in both datasets (Table 2). 

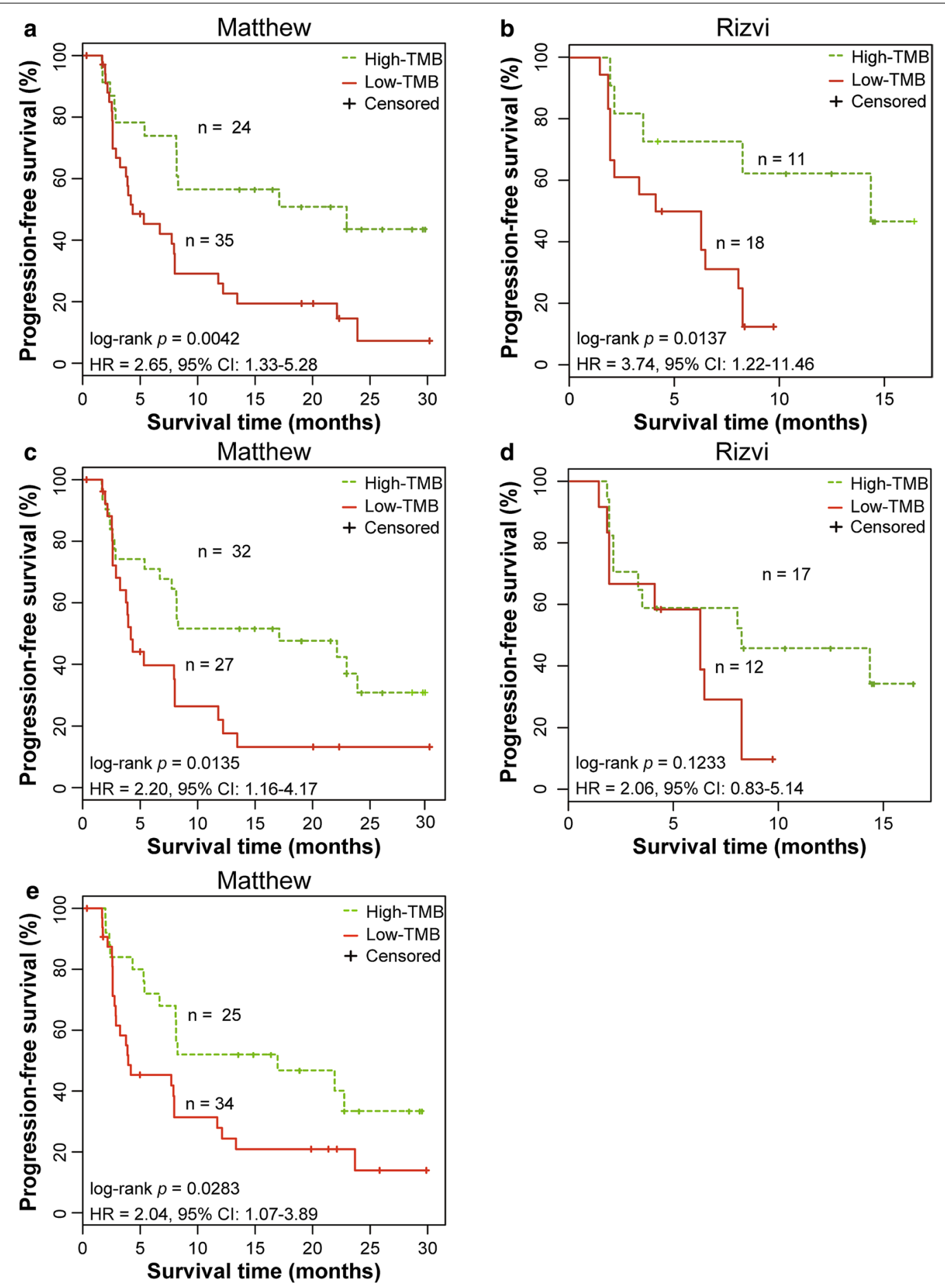

Fig. 4 Performance of other mutation panels for predicting the efficacy of immunotherapy in the test datasets. a Kaplan-Meier curves of progression-free survival (PFS) for 59 advanced lung adenocarcinoma (LUAD) patients in the Matthew dataset, using the 324-gene panel. b KaplanMeier curves of PFS for 29 advanced LUAD patients in the Rizvi dataset, using the 324-gene panel. c Kaplan-Meier curves of PFS for 59 patients in the Matthew dataset, using the 341-gene panel. $\mathbf{d}$ Kaplan-Meier curves of PFS for 29 patients in the Rizvi dataset, using the 341-gene panel. e Kaplan-Meier curves of PFS for 59 patients in the Matthew dataset, using the LUAD-specific 24-gene panel. The $p$ value was determined using log-rank test. The hazard ratio (HR) and 95\% confidence interval (Cl) were determined using univariate Cox regression models 
For the 341-gene mutation panel with a cut-point of $7.40 \mathrm{mut} / \mathrm{Mb}$ [22], containing $6773 \mathrm{CDSs}$ spanning $0.92 \mathrm{Mb}$, its performance in predicting the efficacy of immunotherapy in the Matthew dataset (log-rank $p=0.0135, \mathrm{HR}=2.20,95 \% \mathrm{CI} 1.16-4.17$, Fig. 4c) and the Rizvi dataset (log-rank $p=0.1233, \mathrm{HR}=2.06,95 \%$ CI $0.83-5.14$, Fig. 4 d) was inferior to that of our 106-CDS panel (Table 2).

For the LUAD-specific mutation panel (24-gene panel) with a cut-point of 141 [3], containing 833 CDSs spanning $0.18 \mathrm{Mb}$, univariate survival analyses revealed that the two groups of patients classified by the panel had significantly different PFS after immunotherapy in the Matthew dataset (log-rank $p=0.0283, \mathrm{HR}=2.04,95 \% \mathrm{CI}$ 1.07-3.89, Fig. 4e). However, its performance (HR) was much worse than that of our 106-CDS panel (Table 2). As the Rizvi dataset is the training set to determine the cutpoint (141) of the 24-gene panel in predicting the benefits of pembrolizumab immunotherapy, we did not compare our 106-CDS panel with the 24-gene panel in the dataset, as it is not an independent test data for the 24-gene panel.

\section{Functional characterizations of the 106-CDS panel}

In TCGA dataset, using the 106-CDS panel with a cutpoint of $6.20 \mathrm{mut} / \mathrm{Mb}, 220$ and 266 samples were divided into high- and low-TMB groups, respectively. We found that 7181 genes were differentially expressed between the two groups (Student's t-test, FDR $<0.05$, Fig. 5a, Additional file 5: Table S4), which were significantly enriched in 22 functional pathways (hypergeometric distribution model, FDR $<0.05$, Fig. 5b, Additional file 6: Table S5), including those associated with genomic instability, such as DNA repair [34], DNA replication [35] and chromosome segregation [36]. These results indicated that compared with the low-TMB patients predicted using the 106-CDS panel, the predicted high-TMB patients might have higher genomic instability, thus potentially benefiting from immunotherapy, as they are more likely to harbour neoantigens.

\section{Discussion}

This study describes the generation of a mutation panel comprising $106 \mathrm{CDSs}$ of 100 genes spanning $0.34 \mathrm{Mb}$. Previous studies have reported that the sequencing panel, comprising more than 300 cancer-related genes, can help predict the TMB; however, its performance is apparently low when the number of genes in the panel is lesser than 150 [37]. However, these commercial mutation panels (such as 324-gene and 341-gene panels) were not selected through any feature selection method; thus, their high correlations with the TMB primarily resulted from the

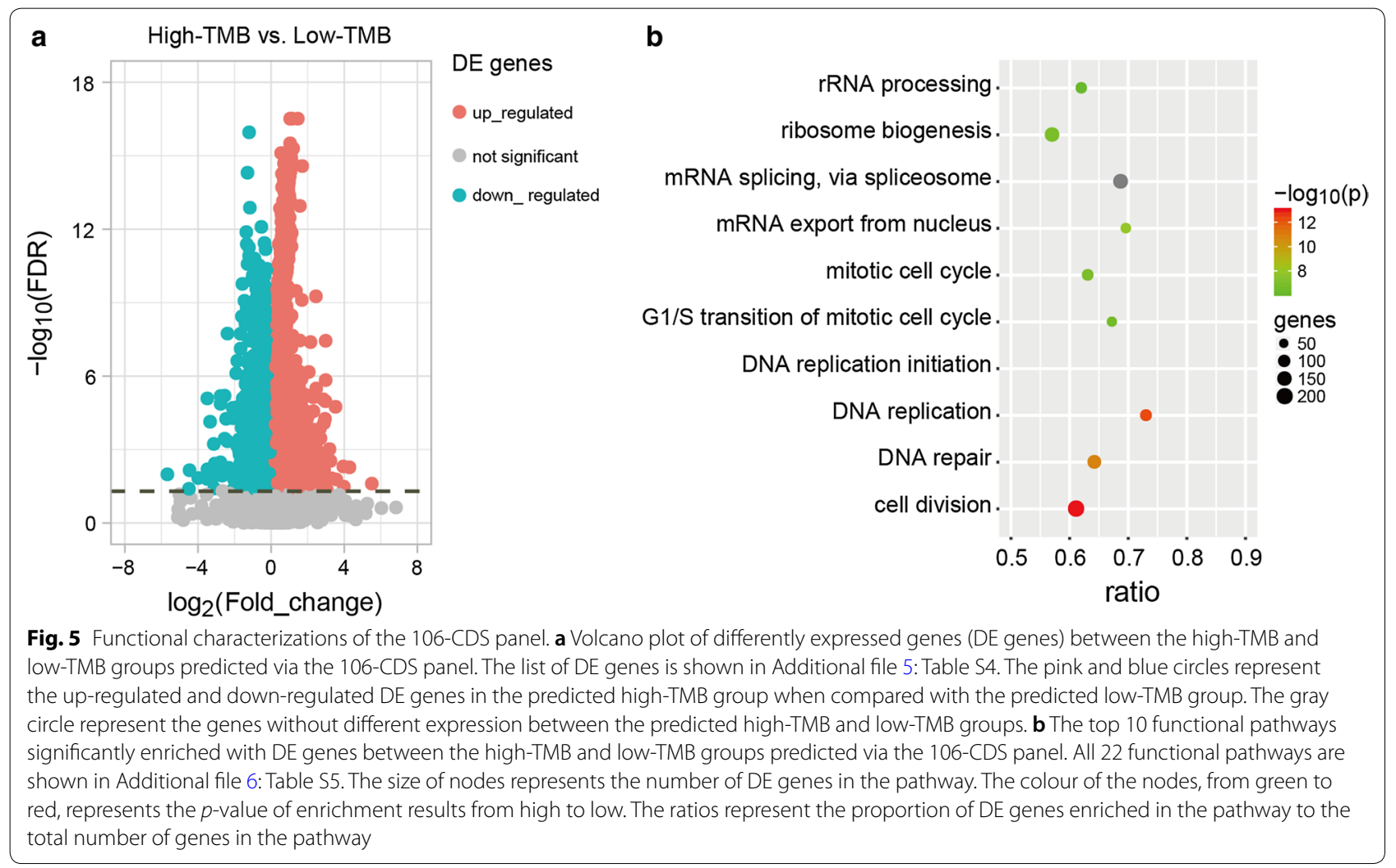


large number of genes included in the panels. In contrast, our 106-CDS mutation panel developed using a genetic algorithm and containing more major variates associated with the TMB is expected to be reliable in estimating the $\mathrm{TMB}$, and its performance was validated in the two independent test datasets. Certain differences in the correlations of our 106-CDS panel and the TMB were observed in the two test datasets, thus potentially accounting for their different sample sizes or sample collections; these correlations require further validation in a large-scale clinical trial.

The present results show that the 106-CDS panel with a cut-point of $6.20 \mathrm{mut} / \mathrm{Mb}$ preferably predicted the efficacy of immunotherapy among advanced-stage LUAD patients. For high-TMB patients predicted via the 106CDS panel with a cut-point of $6.20 \mathrm{mut} / \mathrm{Mb}$, immunotherapy with nivolumab plus ipilimumab improved the 1 -year PFS rate to 0.67 , which was markedly higher than the 1-year PFS rate (0.25) of the predicted low-TMB patients. Similarly, the 1-year PFS rate of the predicted high-TMB patients was 0.61 , being markedly higher than the 1-year PFS rate $(0.13)$ of the predicted lowTMB patients after pembrolizumab treatment. However, we considered that the cut-point of the 106-CDS panel, which was set at a median panel score in training dataset, may not be the optimal threshold for predicting the efficacy of various immunotherapy drugs. In order to assess the effect of specific cut-points for predicting the efficacy of immunotherapy, we additionally set the cutpoints of our CDS panel at upper tertiles $(9.17 \mathrm{mut} / \mathrm{Mb})$ and quartiles $(12.13 \mathrm{mut} / \mathrm{Mb})$ of panel scores in training dataset, respectively, and estimated in the two test datasets. The univariate survival analyses revealed that the 106-CDS panel with the cut-point of the upper quartiles (12.13 mut/Mb) had the optimal predictive performance (log-rank $p=0.0079, \mathrm{HR}=3.81,95 \%$ CI 1.33-10.93, Additional file 7: Figure S1A) than the median (log-rank $p=0.0018, \mathrm{HR}=3.35,95 \%$ CI $1.51-7.42$, Fig. 3a) and upper tertiles (log-rank $p=0.0298, \mathrm{HR}=2.59,95 \% \mathrm{CI}$ 1.07-6.27, Additional file 7: Figure S1B) as cut-pionts for the patients treated with nivolumab plus ipilimumab in the Matthew dataset. While, it had the weakest performance $(\log -\operatorname{rank} p=0.1258, \mathrm{HR}=2.58,95 \%$ CI 0.72-9.21, Additional file 7: Figure S1C) than the median (log-rank $p=0.0020, \mathrm{HR}=5.06,95 \%$ CI 1.63-15.69, Fig. 3c) and upper tertiles (log-rank $p=0.0081, \mathrm{HR}=5.82,95 \% \mathrm{CI}$ 1.33-25.51, Additional file 7: Figure S1D) for the patients treated with pembrolizumab in the Rizvi dataset. These results suggest that the 106-CDS panel with a cut-point of $6.20 \mathrm{mut} / \mathrm{Mb}$ can effectively predict patients potentially benefiting from immunotherapies, but the optimal cut-point for a specific immunotherapy drug needs further exploration in a large-scale clinical trial.
The larger the number of genes included in the mutation panel, the higher the expected correlation with the TMB. Our results show that although the number of genes in the 106-CDS panel is twofold less than that of the 324-gene [19] and 341-gene [22] panels, our 106-CDS panel displayed better performance in predicting the efficacy of immunotherapy. Although the length of the 106-CDS panel $(0.34 \mathrm{Mb})$ was longer than the 24-gene panel $(0.18 \mathrm{Mb})$, its performance was markedly better in predicting the efficacy of immunotherapy. These results indicate that the 106-CDS panel of mutations may have higher antigenicity, which needs further confirmation.

Functional annotation revealed that several genes including TP53 [38], AMER1 [39], and TEX15 [40] in the 106-CDS panel are involved in DNA repair and cell cycle arrest, playing a key role in genomic instability. $\mathrm{DE}$ genes between the two groups classified using the 106-CDS panel with a cut-point of $6.20 \mathrm{mut} / \mathrm{Mb}$ were significantly enriched in several pathways associated with genomic instability, such as DNA repair [34], DNA replication [35], and chromosome segregation [36]. These functional analyses indicate that compared with low-TMB patients predicted using the 106-CDS panel, the high-TMB patients potentially have higher genomic instability and are more likely to harbour neoantigens.

\section{Conclusions}

The CDS mutation panel spanning only $0.34 \mathrm{Mb}$ can effectively predict the efficacy of immunotherapy for LUAD patients through accurate estimation of the TMB. This small panel is preferable for clinical samples because of its low cost and time consumption.

\section{Supplementary information}

Supplementary information accompanies this paper at https://doi. org/10.1186/s12967-019-02199-6.

Additional file 1. The genetic algorithm for searching a CDS panel. Additional file 2: Table S1. Description of the 106-CDS panel. Additional file 3: Table S2. Technical details of TMB evaluation. Additional file 4: Table S3. The correlation of the clinical factors and tumour cell proportions with the tumour mutation burden.

Additional file 5: Table S4. List of differently expressed genes between high-TMB and low-TMB groups predicted via the 106-CDS panel.

Additional file 6: Table S5. The functional pathways enriched with differently expressed genes.

Additional file 7: Figure S1. Performance of 106-CDS panel with the other cut-points for predicting the efficacy of immunotherapy.

\section{Abbreviations}

LUAD: lung adenocarcinomas; NSCLC: non-small cell lung cancer; PD-1: programmed death-1; PD-L1: programmed death-ligand 1; CTLA-4: cytotoxic T lymphocyte-associated antigen 4; IHC: immunohistochemistry; TMB: tumour mutation burden; WES: whole-exome sequencing; CDSs: coding sequences; 
PFS: progression-free survival; TCGA: The Cancer Genome Atlas; FDR: false discovery rate; HRs: hazard ratios; Cls: confidence intervals; GO: Gene Ontology; DE genes: differentially expressed genes; FDA: Food and Drug Adminstration.

\section{Acknowledgements}

None.

\section{Authors' contributions}

LSQ conceived and designed the experiments, and wrote the manuscript. LA designed analytical methods and wrote the manuscript. ZG conceived the idea. $Y L$ and WBJ designed and performed the experiments. MYL processed and analyzed the data. XL and ZYZ performed the experiments. SNZ and YXL collected and processed the data. WYZ and YYG interpreted data. All authors read and approved the final manuscript.

\section{Funding}

This work was supported by National Natural Science Foundation of China (Grant numbers: 61701143, 81872396 and 61673143), the Joint Scientific and Technology Innovation Fund of Fujian Province (Grant number: 2016Y9044).

\section{Availability of data and materials}

The datasets of this article were generated from the TCGA database and two articles published by Rizvi et al. [4] and Matthew et al. [5].

\section{Ethics approval and consent to participate}

Not applicable.

\section{Consent for publication}

Not applicable.

\section{Competing interests}

The authors declare that they have no competing interests.

\section{Author details}

${ }^{1}$ College of Bioinformatics Science and Technology, Harbin Medical University, Harbin 150086, China. ${ }^{2}$ Department of Bioinformatics, Key Laboratory of Ministry of Education for Gastrointestinal Cancer, School of Basic Medical Sciences, Fujian Medical University, Fuzhou 350001, China. ${ }^{3}$ Key Laboratory of Medical Bioinformatics, Fujian Province, Fuzhou 350001, China.

Received: 31 May 2019 Accepted: 26 December 2019

Published online: 14 January 2020

\section{References}

1. Chansky K, Sculier J-P, Crowley JJ, Giroux D, Van Meerbeeck J, Goldstraw P, et al. The International Association for the Study of Lung Cancer Staging Project: prognostic factors and pathologic TNM stage in surgically managed non-small cell lung cancer. J Thorac Oncol. 2009;4:792-801.

2. Siegel RL, Miller KD, Jemal A. Cancer statistics. CA Cancer J Clin. 2015;65:5-29.

3. Lyu G-Y, Yeh Y-H, Yeh Y-C, Wang Y-C. Mutation load estimation model as a predictor of the response to cancer immunotherapy. NPJ Genomic Med. 2018;3:12.

4. Rizvi NA, Hellmann MD, Snyder A, Kvistborg P, Makarov V, Havel JJ, et al. Cancer immunology. Mutational landscape determines sensitivity to PD-1 blockade in non-small cell lung cancer. Science. 2015;348:124-8.

5. Hellmann MD, Nathanson T, Rizvi H, Creelan BC, Sanchez-Vega F, Ahuja $A$, et al. Genomic features of response to combination immunotherapy in patients with advanced non-small-cell lung cancer. Cancer Cell. 2018;33(843-852):e4.

6. Chalmers ZR, Connelly CF, Fabrizio D, Gay L, Ali SM, Ennis R, et al. Analysis of 100,000 human cancer genomes reveals the landscape of tumor mutational burden. Genome Med. 2017;9:34.

7. Tawbi HA, Forsyth PA, Algazi A, Hamid O, Hodi FS, Moschos SJ, et al. Combined nivolumab and ipilimumab in melanoma metastatic to the brain. $\mathrm{N}$ Engl J Med. 2018;379:722-30.

8. Reck M, Rodríguez-Abreu D, Robinson AG, Hui R, Csőszi T, Fülöp A, et al. Pembrolizumab versus chemotherapy for PD-L1-positive non-smallcell lung cancer. N Engl J Med. 2016;375:1823-33.
9. Hui R, Garon EB, Goldman JW, Leighl NB, Hellmann MD, Patnaik A, et al. Pembrolizumab as first-line therapy for patients with PD-L1-positive advanced non-small cell lung cancer: a phase 1 trial. Ann Oncol. 2017;28:874-81.

10. Carbone DP, Reck M, Paz-Ares L, Creelan B, Horn L, Steins M, et al. Firstline nivolumab in stage IV or recurrent non-small-cell lung cancer. $N$ Engl J Med. 2017;376:2415-26.

11. Garon EB. Cancer immunotherapy trials not immune from imprecise selection of patients. N Engl J Med. 2017;376:2483-5.

12. Brunnström $H$, Johansson $A$, Westbom-Fremer S, Backman M, Djureinovic D, Patthey A, et al. PD-L1 immunohistochemistry in clinical diagnostics of lung cancer: inter-pathologist variability is higher than assay variability. Mod Pathol. 2017;30:1411-21.

13. Koeppel F, Blanchard S, Jovelet C, Genin B, Marcaillou C, Martin E, et al. Whole exome sequencing for determination of tumor mutation load in liquid biopsy from advanced cancer patients. PLOS ONE. 2017;12:e0188174-e0188174.

14. Hendriks LE, Rouleau E, Besse B. Clinical utility of tumor mutational burden in patients with non-small cell lung cancer treated with immunotherapy. Transl lung cancer Res. 2018;7:647-60.

15. Ready N, Hellmann MD, Awad MM, Otterson GA, Gutierrez M, Gainor $J F$, et al. First-line nivolumab plus ipilimumab in advanced non-smallcell lung cancer (CheckMate 568): outcomes by programmed death ligand 1 and tumor mutational burden as biomarkers. J Clin Oncol. 2019;37:992-1000.

16. Teixidó C, Vilariño N, Reyes R, Reguart N. PD-L1 expression testing in non-small cell lung cancer. Ther Adv Med Oncol. 2018;10:1758835918763493.

17. Meléndez B, Van Campenhout C, Rorive S, Remmelink M, Salmon I, D'Haene N. Methods of measurement for tumor mutational burden in tumor tissue. Transl lung cancer Res. 2018;7:661-7.

18. Allgäuer M, Budczies J, Christopoulos P, Endris V, Lier A, Rempel E, et al. Implementing tumor mutational burden (TMB) analysis in routine diagnostics-a primer for molecular pathologists and clinicians. Transl lung cancer Res. 2018;7:703-15.

19. Hellmann MD, Ciuleanu T-E, Pluzanski A, Lee JS, Otterson GA, AudigierValette $C$, et al. Nivolumab plus ipilimumab in lung cancer with a high tumor mutational burden. N Engl J Med. 2018;378:2093-104.

20. Frampton GM, Fichtenholtz A, Otto GA, Wang K, Downing SR, He J, et al. Development and validation of a clinical cancer genomic profiling test based on massively parallel DNA sequencing. Nat Biotechnol. 2013:31:1023-31.

21. Yarchoan M, Hopkins A, Jaffee EM. Tumor mutational burden and response rate to PD-1 inhibition. N Engl J Med. 2017;377:2500-1.

22. Rizvi H, Sanchez-Vega F, La K, Chatila W, Jonsson P, Halpenny D, et al. Molecular determinants of response to anti-programmed cell death (PD)-1 and anti-programmed death-ligand 1 (PD-L1) blockade in patients with non-small-cell lung cancer profiled with targeted nextgeneration sequencing. J Clin Oncol. 2018;36:633-41.

23. Cheng DT, Mitchell TN, Zehir A, Shah RH, Benayed R, Syed A, et al. Memorial sloan kettering-integrated mutation profiling of actionable cancer targets (MSK-IMPACT): a hybridization capture-based next-generation sequencing clinical assay for solid tumor molecular oncology. J Mol Diagn. 2015;17:251-64.

24. Heemskerk B, Kvistborg P, Schumacher TNM. The cancer antigenome. EMBO J. 2013;32:194-203.

25. Lawrence MS, Stojanov P, Polak P, Kryukov GV, Cibulskis K, Sivachenko $A$, et al. Mutational heterogeneity in cancer and the search for new cancer-associated genes. Nature. 2013;499:214-8.

26. Garofalo A, Sholl L, Reardon B, Taylor-Weiner A, Amin-Mansour A, Miao $D$, et al. The impact of tumor profiling approaches and genomic data strategies for cancer precision medicine. Genome Med. 2016;8:79.

27. Network CGAR. Comprehensive molecular profiling of lung adenocarcinoma. Nature. 2014;511:543-50.

28. Havel JJ, Bruggeman C, Wong P, Moreira AL, Merghoub T, Garon EB, et al. Mutational landscape determines sensitivity to PD-1 blockade in non-small cell lung cancer. Science (80-). 2015;348:124-8.

29. Yu J, Wu WKK, Li X, He J, Li X-X, Ng SSM, et al. Novel recurrently mutated genes and a prognostic mutation signature in colorectal cancer. Gut. 2015;64:636-45. 
30. Qi L, Li T, Shi G, Wang J, Li X, Zhang S, et al. An individualized gene expression signature for prediction of lung adenocarcinoma metastases. Mol Oncol. 2017;11:1630-45.

31. Benjamini Y, Hochberg Y. Controlling the False Discovery Rate: A Practical and Powerful Approach to Multiple Testing. J R Stat Soc Ser B. 1995;57:289-300.

32. Schneider A, Hommel G, Blettner M. Linear regression analysis: part 14 of a series on evaluation of scientific publications. Dtsch Arztebl Int. 2010:107:776-82.

33. Bland JM, Altman DG. The logrank test. BMJ. 2004;328:1073.

34. Kastan MB, Bartek J. Cell-cycle checkpoints and cancer. Nature. 2004;432:316-23.

35. Maya-Mendoza A, Moudry P, Merchut-Maya JM, Lee M, Strauss R, Bartek $J$. High speed of fork progression induces DNA replication stress and genomic instability. Nature. 2018;559:279-84.

36. Zhong A, Chen P-L, Chen Y, Chang M, Gau R, Riley DJ, et al. Aberrant DNA damage response and dna repair pathway in high glucose conditions. $J$ Can Res Updates. 2018;7:64-74.
37. Campesato LF, Barroso-Sousa R, Jimenez L, Correa BR, Sabbaga J, Hoff $\mathrm{PM}$, et al. Comprehensive cancer-gene panels can be used to estimate mutational load and predict clinical benefit to PD-1 blockade in clinical practice. Oncotarget. 2015;6:34221-7.

38. Vogelstein B, Kinzler KW. p53 function and dysfunction. Cell. 1992;70:523-6.

39. Kim MKH, Min DJ, Rabin M, Licht JD. Functional characterization of Wilms tumor-suppressor WTX and tumor-associated mutants. Oncogene. 2010;30:832.

40. Lin X, Chen Z, Gao P, Gao Z, Chen H, Qi J, et al. TEX15: a DNA repair gene associated with prostate cancer risk in Han Chinese. Prostate. 2017:77:1271-8.

\section{Publisher's Note}

Springer Nature remains neutral with regard to jurisdictional claims in published maps and institutional affiliations.
Ready to submit your research? Choose BMC and benefit from:

- fast, convenient online submission

- thorough peer review by experienced researchers in your field

- rapid publication on acceptance

- support for research data, including large and complex data types

- gold Open Access which fosters wider collaboration and increased citations

- maximum visibility for your research: over $100 \mathrm{M}$ website views per year

At BMC, research is always in progress.

Learn more biomedcentral.com/submissions 